\title{
MEK1 and MEK2 isoforms regulate distinct functions in pancreatic cancer cells
}

\author{
LEI ZHOU ${ }^{1 *}$, XIAODONG TAN $^{1 *}$, HIDENOBU KAMOHARA $^{2}$, WEI WANG ${ }^{1}$, BAOSHENG WANG $^{1}$, \\ JINGANG LIU $^{1}$, HIROSHI EGAMI ${ }^{3}$, HIDEO BABA ${ }^{2}$ and XIANWEI DAI ${ }^{1}$ \\ ${ }^{1}$ Department of Hepato-pancreato-biliary Tumour Surgery, China Medical University Shengjing Hospital, \\ Shenyang, P.R. China; ${ }^{2}$ Department of Gastroenterological Surgery, Graduate School of Medical \\ Sciences, Kumamoto University; ${ }^{3}$ Department of Surgery, Ugatake Hospital, Kumamoto, Japan
}

Received December 7, 2009; Accepted February 22, 2010

DOI: $10.3892 /$ or_00000853

\begin{abstract}
The mitogen-activated protein kinase kinase 1/2 (MEK1/2) signalling pathway plays a central role in tumour progression. Small molecules that inhibit MEK1/2 are therefore considered attractive candidates for anti-cancer drugs. However, the exact contributions of MEK1 and MEK2 to the development of pancreatic cancer remain to be established. To differentiate the functions of MEK1 and MEK2 in a cultured pancreatic cancer cell line, we utilised shRNAmediated knockdown of their two mRNAs individually. We studied the effects of MEK1 and MEK2 knockdown on cell morphology, proliferation, mitotic arrest, and in vitro invasion capability in PC-1.0 cells. The results showed that inhibition of MEK1 expression was an effective and specific approach to inhibit cell proliferation and induce $G_{0} / G_{1}$ arrest. On the other hand, MEK2 knockdown specially altered cell morphology and inhibited the invasive ability of pancreatic cancer cells. Therefore, MEK1 and MEK2 mediate different biological responses in cultured pancreatic cancer cells. These proteins could become distinct targets for the inhibition of specific cellular functions in the treatment of pancreatic cancer.
\end{abstract}

\section{Introduction}

By the time it is diagnosed, pancreatic cancer has often undergone extensive local invasion and/or metastasis,

Correspondence to: Dr Xiaodong Tan, Department of Hepatopancreato-biliary Tumour Surgery, China Medical University Shengjing Hospital, No. 36, Sanhao Street, Heping District, Shenyang 110004, P.R. China

E-mail: tanxd@hotmail.com

*Contributed equally

Key words: pancreatic cancer, mitogen-activated protein kinase kinase 1, MEK2, RNAi, function regulation precluding a therapeutic surgical solution. Development of new molecular therapeutic methods targeting the invasion/ metastasis-related factors is a promising avenue for improving the prognosis of patients with pancreatic cancer. However, the cellular and molecular mechanisms of invasion/metastasis of pancreatic cancer have not been fully elucidated.

Two hamster pancreatic cancer cell lines with different potentials for invasion and metastasis after intra-pancreatic transplantation, PC-1 (low potential) and PC-1.0 (high potential), were established from a pancreatic ductal carcinoma induced by N-nitrosobis (2-oxopropyl) amine (BOP) in a Syrian golden hamster (1-3). In our previous study, gene expression differences between PC-1.0 and PC-1 cells were examined using the representational difference analysis (RDA) method. We identified mitogen-activated protein kinase kinase 2 (MEK2) as a factor that was correlated with the invasion and metastasis potential of these cell lines (4). Further investigation confirmed that MEK2 was involved in invasion/metastasis of both hamster and human pancreatic cancer cells (5).

MEK2 is a key kinase in the mitogen-activated protein kinase (MAPK) signal transduction pathway, which is involved in many cellular processes, including cell proliferation, differentiation, cell division, stress, and apoptosis (6-9). The MAPK pathways are evolutionarily conserved in all eukaryotes and can be organized into a three-kinase hierarchy: a MAPK, a MAPK activator (MAP kinase kinase, MKK or MEK), and a MAP kinase kinase activator (MAP kinase kinase kinase, MAPKKK) (10). MEK1 and MEK2 are the only two isoforms of the MEK family and are commonly referred to as MEK1/2. Their amino acid sequences are $\sim 85 \%$ identical and they are expressed ubiquitously in cell lines and tissues. Although it is commonly assumed that the two isoforms are functionally equivalent, several lines of evidence indicate that they are regulated differentially and may exert non-redundant functions (11-13). Thus far, the individual roles of MEK1 and MEK2 in pancreatic cancer cells remain to be explored.

In this study, short-hairpin RNAs (shRNAs) expressed in a retroviral vector were used to specifically silence the expressions of MEK1 and MEK2 in the highly invasive and metastatic pancreatic cancer cell line PC-1.0 to demonstrate the distinct functions of regulation by MEK1 and MEK2 in pancreatic cancer cells. 


\section{Materials and methods}

Cell lines and cell culture. Two hamster pancreatic cancer cell lines, the weakly invasive and metastatic cell line PC-1 and the highly invasive and metastatic cell line PC-1.0 were used. The PC-1 cell line was established from pancreatic ductal/ductular adenocarcinomas induced by BOP in a Syrian golden hamster (1). The PC-1.0 cell line was established from a subcutaneous tumour produced after inoculation of PC-1 cells (2). These two cell lines exhibited differential growth morphology in vitro. The PC-1 cells formed island-like cell colonies, and PC-1.0 cells mainly grew as single cells (3).

Subclones of the PC-1.0 cells that expressed the MEK 1 or MEK2 shRNA, as well as GP2-293 packaging cells (for production of retrovirus), were used. These cell lines were incubated in RPMI-1640 (Gibco-BRL, Grand Island, NY), supplemented with $10 \%$ foetal bovine serum (Bioserum, Victoria, Australia), $100 \mathrm{U} / \mathrm{ml}$ penicillin $\mathrm{G}$, and $100 \mu \mathrm{g} / \mathrm{ml}$ streptomycin at $37^{\circ} \mathrm{C}$ in a humidified atmosphere of $5 \% \mathrm{CO}_{2}$. The cells were serum-starved overnight before experiments.

Antibodies. Rabbit polyclonal antibodies raised against epitopes of human MEK1, MEK2, and ß-actin (Santa Cruz Biotechnology, Santa Cruz, CA) were used as primary antibodies. Horseradish peroxidase-conjugated secondary antibodies and FITC-labelled fluorescent antibodies (Santa Cruz Biotechnology) were used as secondary antibodies for Western blotting.

Design of anti-MEK1 and anti-MEK2 RNAi sequence and construction of shRNA-expressing retroviral vector. The MEK1- and MEK2-specific target sequences were chosen according to the comprehensive online RNAi tool (Clontech Laboratories, Inc., CA, USA) at http://bioinfo2.clontech. com/rnaidesigner using the MEK1 (Gene Bank Accession no.: NM_002755) and MEK2 (Gene Bank Accession no.: NM_030662) reference sequences. The MEK1 shRNA targeted nt 614-632 of the mRNA (5'-GGAGAAGCACAAGAUC AUG-3') and the MEK2 shRNA targeted nt 1216-1234 (5'-CCUGGACUAUAUUGUGAAC-3') of the mRNA. The shRNAs were chemically synthesized and cloned into the retroviral vector pSIREN-RetroQ-Zsgreen as described previously (14). Sequencing of the vectors to confirm shRNA insertion was performed in an ABI Prism 3100 genetic analyzer with a BigDye Terminator sequencing kit according to the manufacturer's instructions (Applied Biosystems, CA, USA).

Production of retroviruses and retroviral infection. GP2-293 cells $\left(8 \times 10^{5}\right)$ were cotransfected with the packaging vector pVSV-G and RNAi-Ready pSIREN-RetroQ-Zsgreen vector containing the MEK1 or MEK2 shRNA inserts (Clontech Laboratories, Inc.) using Lipofectamine 2000 (Invitrogen, Carlsbad, CA, USA). Control cell lines were generated by infecting GP2-293 cells with the appropriate empty control vectors (RNAi-Ready pSIREN-RetroQ-Zsgreen vector and pVSV-G vector). After transfection, cells were cultured for $48 \mathrm{~h}$ in RPMI-1640. The retrovirus-containing supernatants were filtered and used to infect PC- 1.0 cells according to the manufacturer's recommendations of Retroviral Gene Transfer and Expression User Manual (PT3132, Clontech Laboratories, Inc.). For stable silencing of MEK1 and MEK2, the colonies of the infected PC-1.0 cells were picked under a fluorescent microscope, expanded, and analysed separately.

Analysis of MEK1 and MEK2 mRNA expressions by RT$P C R$. Total RNA was isolated from the MEK1 or MEK2 shRNA-expressing PC-1.0 cells and the empty vector-infected control PC- 1.0 cells. An aliquot of $1 \mu \mathrm{g}$ of total RNA from each sample was reverse-transcribed to cDNA using the SuperScript II kit (Life Technologies, Inc.) as previously described (5). The specific PCR primer sequences for MEK1, MEK2 and B-actin were: MEK1 forward: 5'-ATTATTGTTC CCCTAAGTGGATTG-3', reverse: 5'-TTACAACAGCATT GGTACTTGGAT-3'; MEK2 forward: 5'-GCAGTCGGACA TCTGGAGCA-3', reverse: 5'-CACCGTTG GGCAGCTTA GGA-3'; ß-actin forward: 5'-GTGGGGCGCCCCAGGCA CCA-3', reverse: 5'-CTCCTTAAGTCACGCACGATTCC-3'. $ß$-actin was used as a normalising control. After an initial denaturation step of $95^{\circ} \mathrm{C}$ for $5 \mathrm{~min}$, the PCR was run for 30 cycles of $94^{\circ} \mathrm{C}$ for $30 \mathrm{sec}, 55^{\circ} \mathrm{C}$ for $30 \mathrm{sec}$, and $72^{\circ} \mathrm{C}$ for $1 \mathrm{~min}$, with a final extended step at $72^{\circ} \mathrm{C}$ for $7 \mathrm{~min}$. Independent experiments were done in triplicate. For mRNA quantification, samples were normalised against the expression of $B$-actin mRNA and analysed using GeneSnap and GeneTools software (Syngene, Cambridge, UK).

Analysis of MEK1 and MEK2 protein levels by Western blotting. Cells were grown in 90-mm dishes containing $10 \mathrm{ml}$ of RPMI-1640 plus $10 \%$ foetal bovine serum. The cells were lysed in $1 \mathrm{ml}$ ice-cold RIPA buffer (50 mM Tris, $150 \mathrm{mM}$ $\mathrm{NaCl}, 1 \% \mathrm{NP}-40,0.5 \%$ sodium deoxycholate, $0.1 \%$ SDS, pH 7.5 with $1 \mathrm{mM}$ phenylmethylsulfonyl fluoride, $1 \mathrm{mg} / \mathrm{ml}$ leupeptin, and $1 \mathrm{mg} / \mathrm{ml}$ aprotinin were added immediately before use) on ice for $15 \mathrm{~min}$. After centrifugation (5 min at $5000 \mathrm{rpm})$ at $4^{\circ} \mathrm{C}$, the supernatants of cell lysates were collected and stored at $-80^{\circ} \mathrm{C}$. $\beta$-actin was detected as a loading control.

Western blotting was performed as described previously (15). In brief, samples of equivalent total protein $(20 \mu \mathrm{g})$ were run in a $5 \%$ polyacrylamide slab gel and transferred to a polyvinylidene fluoride (PVDF) membrane (Bio-Rad, Anaheim, USA). The membranes were incubated with primary antibody diluted in $0.1 \%$ Tween-20/PBS overnight at $4{ }^{\circ} \mathrm{C}$. The blots were then incubated with horseradish peroxidase-conjugated secondary antibody (diluted 1:5000) in $0.1 \%$ Tween-20/PBS. Enhanced chemiluminescence (Santa Cruz Biotechnology) was used to detect the signals, which were developed on Kodak scientific imaging film (Eastman Kodak Company, Rochester, NY).

In vitro proliferation assay. The MEK1-knockdown, MEK2knockdown, and control PC-1.0 cells were seeded at a density of 5000 cells per well in 96-well plates in culture medium containing 10\% FBS. After 3 days, the number of viable cells was counted using the Cell counting kit 8 (Dojindo Co., Kumamoto, Japan) according to the manufacturer's instructions. The assay reagent is a tetrazolium compound (WST-x 8) that is reduced by live cells into a coloured formazan product that can be measured at $450 \mathrm{~nm}$. The 


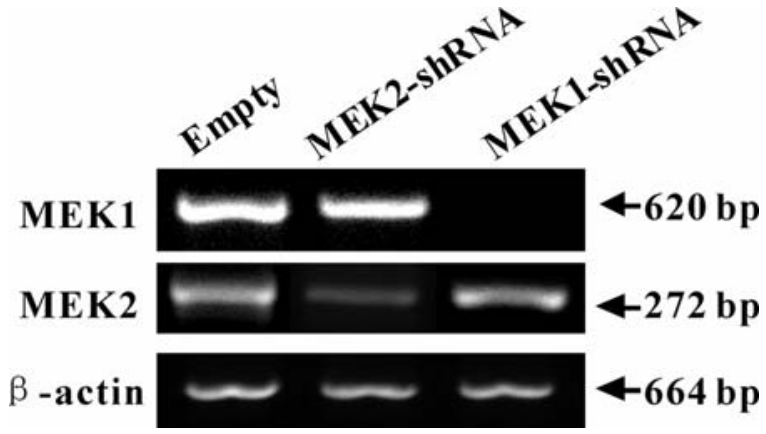

Figure 1. MEK1 and MEK2 mRNA expression in PC-1.0 cells after shRNAmediated knockdown. Expression of MEK1 shRNAs in PC-1.0 cells significantly decreased MEK1 mRNA levels compared with the empty vector but showed no inhibition of MEK2 expression. Similarly, MEK2 shRNAs markedly decreased MEK2 mRNA levels but did not affect MEK1 expression. B-actin served as the normalisation control.

quantity of formazan product measured at $450 \mathrm{~nm}$ is directly proportional to the number of live cells in the culture. The experiments were repeated in triplicate wells.

Cell cycle analysis. Analyses of the cell cycle and mitotic arrest were performed using propidium iodide (PI) to visualise DNA. The cells were trypsinised, pelleted, and resuspended in one volume of ice-cold phosphate-buffered saline (PBS). The suspension was gently agitated while three volumes of ice-cold 95\% ethanol were slowly added. Cold reagents and the gradual addition of ethanol were used to reduce clumping of cells. The cells were then pelleted and resuspended in equal volumes of $30 \mu \mathrm{g} / \mathrm{ml} \mathrm{PI}$ and $100 \mu \mathrm{g} / \mathrm{ml}$ RNase A, both in PBS. Stained cells were stored overnight at $4^{\circ} \mathrm{C}$ and protected from light until analysis. Cell cycle was analysed using a FACScan flow cytometer (Becton Dickinson, San Jose, CA) to determine the percentage of cells in the $\mathrm{G} 1, \mathrm{G} 2$, and $\mathrm{S}$ phases.

In vitro invasion assay. The in vitro invasion assay was done using Invasion Chambers (Becton Dickinson Labware, Bedford, MA). RPMI-1640 medium (1 ml) with 10\% FBS and, as a chemoattractant, the same volume of serum-free conditioned medium of BALB/3T3 cells were added to the cluster plate well. Cell suspension $(500 \mu 1)\left(1 \times 10^{5} / \mathrm{ml}\right)$ of MEK1-knockdown, MEK2-knockdown, or control PC-1.0 cells in RPMI-1640 medium with $10 \%$ FBS and the same volume of test materials dialysed to PBS were added to the inside of the transwell. These cells were incubated for $12 \mathrm{~h}$ at $37^{\circ} \mathrm{C}$. After incubation, cells remaining in the upper surface of the filter were wiped off with a cotton swab, and cells that had migrated to the lower side of the filter were fixed and stained with Diff-Quik reagent (Dade Behring, Dugen, Switzerland). The number of cells migrating to the lower side of the filter was determined by counting the number of nuclei within an area of $1 \mathrm{~mm}^{2}$ on the grid at a magnification of $\mathrm{x} 100$.

Statistical analysis. The data are expressed as the mean values \pm SD unless otherwise stated. The differences between each group were evaluated by unpaired Student's t-test with the Stat View program (SAS Institute, Inc., Cary, NC). $\mathrm{P}<0.05$ was considered significant.

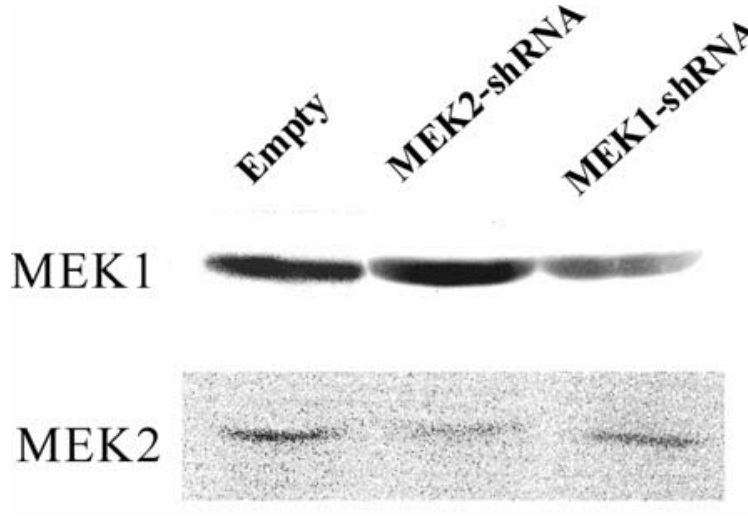

Figure 2. MEK1 and MEK2 protein levels in PC-1.0 cells after MEK1 or MEK2 knockdown. MEK1 shRNA expression in PC-1.0 cells significantly decreased MEK1 protein levels compared with the empty vector but had no effect on MEK2 expression. MEK2 shRNA expression markedly decreased MEK2 protein levels but had no effect on MEK1 protein. B-actin was detected as a loading control.

\section{Results}

Construction of MEK1-shRNA and MEK2-shRNA plasmids. To perform cell-function experiments, we employed pSIRENRetroQ-Zsgreen vectors containing a MEK1 or MEK2 shRNA to block MEK1 or MEK2 expression. PC-1.0 cells infected with the control retrovirus exhibited cell morphology, cell proliferation, cell cycle, and in vitro invasion comparable to those observed in non-infected PC-1.0 cells, suggesting that the vectors themselves do not affect the biological function of PC-1.0 cells (data not shown). To block endogenous MEK1 or MEK2, we tested three shRNA sequences against each gene in the RNAi-Ready pSIREN-RetroQ-Zsgreen vector. We found that all three shRNAs targeting either gene lowered endogenous MEK1 or MEK2 mRNA levels, respectively, with maximum reductions of $98.4 \%$ (MEK1-shRNA 3) and $77.5 \%$ (MEK2-shRNA 3) (Fig. 1). The MEK1-shRNA 3 and MEK2-shRNA 3 vectors also reduced MEK1 and MEK2 protein levels, respectively, compared with the control vectors (Fig. 2). Thus, these two vectors that achieved the greatest degree of knockdown were selected for the following experiments.

Cellular morphology and growth patterns in MEK1-and MEK2-knockdown PC-1.0 cells. We found that the MEK2knockdown PC-1.0 cells grew in an aggregated or clumped pattern in culture. However, MEK1-knockdown cells showed no change in growth pattern compared with that of empty vector-infected PC-1 cells (Fig. 3).

Effects of MEK1 and MEK2 knockdown on proliferation of $P C-1.0$ cells. We investigated the effects of MEK1 and MEK2 knockdown on cell proliferation in $\mathrm{PC}-1.0$ pancreatic cancer cells. We found that down-regulation of MEK1 inhibited proliferation and growth of PC-1.0 cells (Fig. 4). In particular, 48 and $72 \mathrm{~h}$ after incubation, PC-1.0 cells showed markedly decreased proliferative activity, with inhibition rates of 62.0 and $60.5 \%$, respectively, compared with the control cells $(\mathrm{P}<0.05)$. On the other hand, there was no statistically significant difference in the growth of PC- 1.0 cells after 

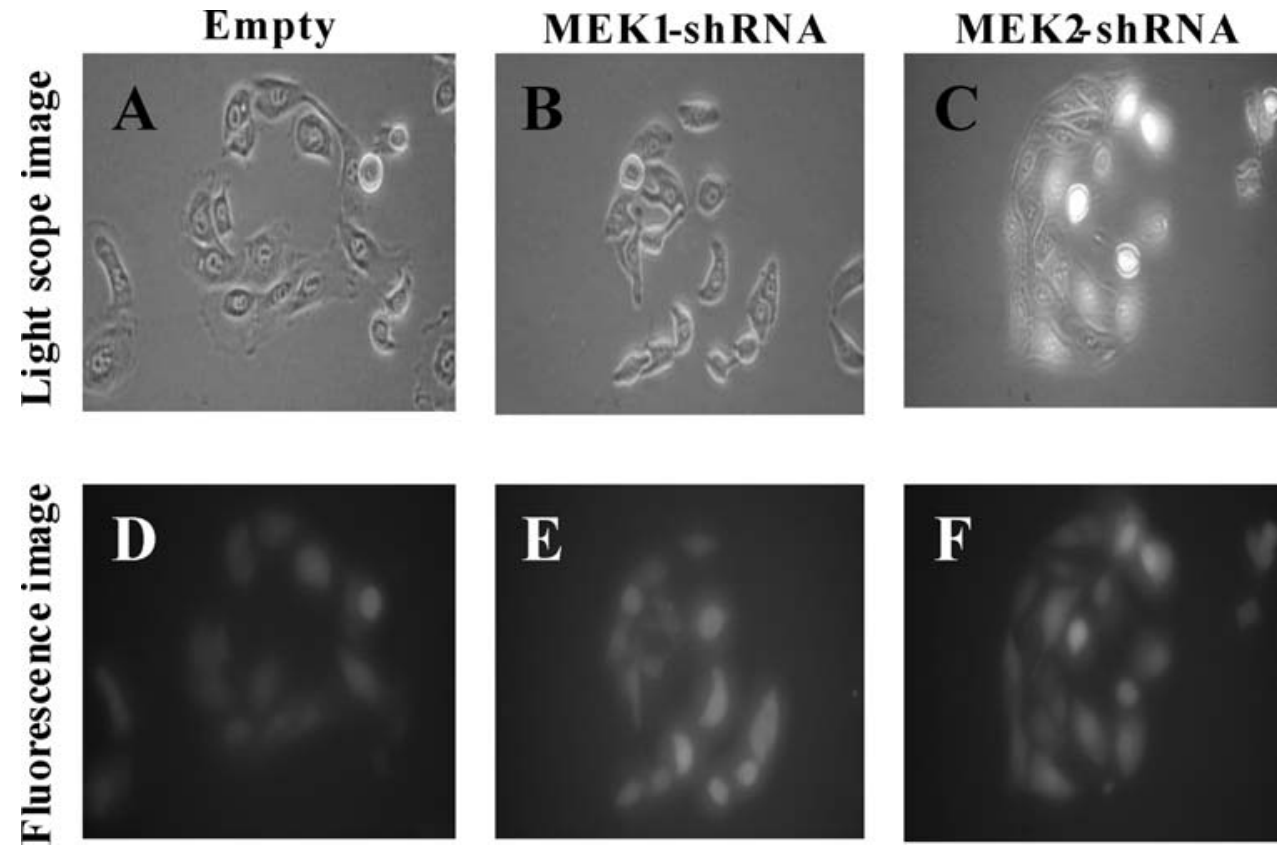

Figure 3. Cell morphology of MEK1-knockdown and MEK2-knockdown PC-1.0 cells. MEK2 knockdown apparently induced cell aggregation in PC-1.0 cells (C) compared with the control cells (A). In contrast, MEK1 knockdown showed no significant effect on cell growth patterns in PC-1.0 cells (B). The fluorescent images show stable transfection of empty control vector (D), MEK1-shRNA (E), and MEK2-shRNA (F).

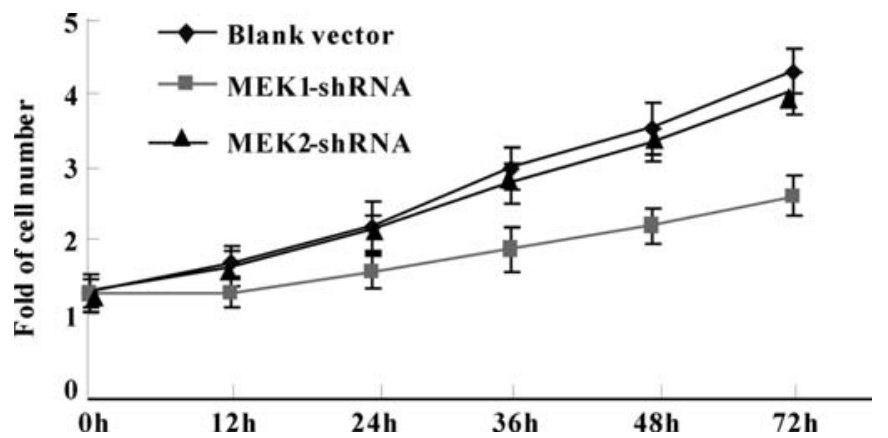

Figure 4. Cell proliferation in MEK1- and MEK2-knockdown PC-1.0 cells. Knockdown of MEK1 exerted an apparent anti-proliferative effect on PC1.0 cells after 48 and $72 \mathrm{~h}$. On the contrary, MEK2 knockdown exhibited no significant inhibition of cell growth or proliferation in PC-1.0 cells.

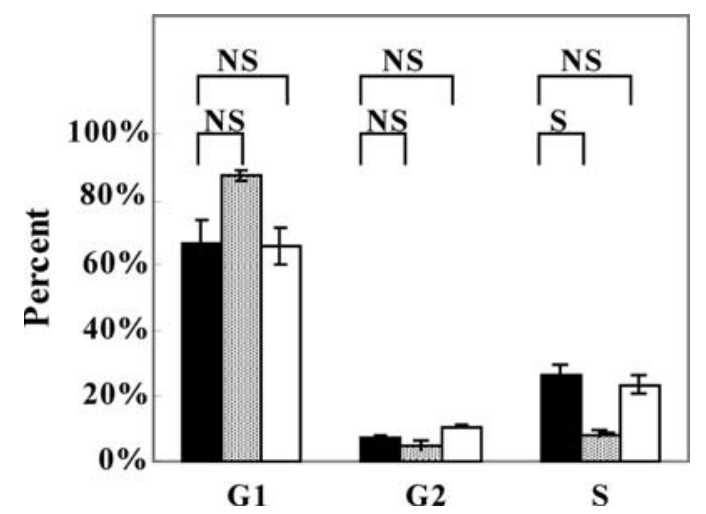

Figure 5. Effects of MEK1 and MEK2 knockdown on mitotic arrest in PC1.0 cells. MEK1 knockdown decreased the percentage of cells in S phase, but no change to the cell cycle was observed in MEK2-knockdown PC-1.0 cells. Black bar, control PC-1.0 cells with empty vector; dotted bar, MEK1knockdown PC-1.0 cells; white bar, MEK2-knockdown PC-1.0 cells. S, significant; NS, not significant.

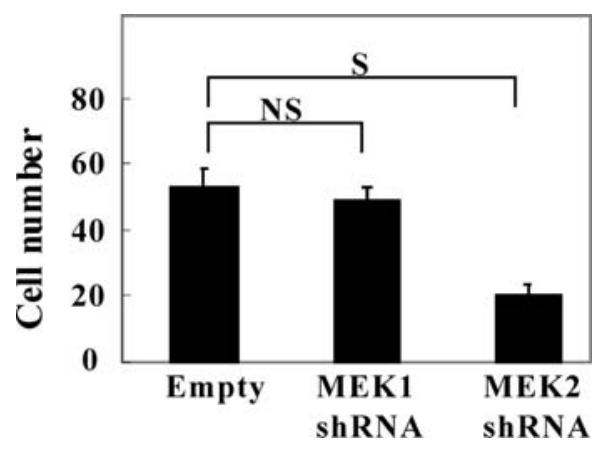

Figure 6. Invasive capability in MEK1- and MEK2-knockdown PC-1.0 cells. Knockdown of MEK2 significantly inhibited the invasive capability of PC-1.0 cells compared with cells expressing the empty vector. However, MEK1 knockdown did not affect invasive capability. S, significant; NS, not significant.

down-regulation of MEK2 gene expression compared with the control cells $(\mathrm{P}>0.05)$.

Effects of MEK1 and MEK2 knockdown on cell cycle phase in PC-1.0 cells. The cell cycle distributions of MEK1knockdown and MEK2-knockdown PC-1.0 cells were analysed using a FACScan flow cytometer. In MEK1knockdown cells, we found only $8.1 \pm 0.7 \%$ of cells in the $\mathrm{S}$ phase of mitosis. In contrast, $26.6 \pm 5.4 \%$ of control PC-1.0 cells were in $\mathrm{S}$ phase $(\mathrm{P}<0.05$, Fig. 5), and there was no significant difference between the MEK2-knockdown cells and the control cells ( $\mathrm{P}>0.05$, Fig. 5).

Effects of MEK1 and MEK2 knockdown on in vitro invasion in PC-1.0 cells. As shown in Fig. 6, the control cells exhibited a strong invasive capability (invasive cell number $=52.6 \pm 5.8$ ). 
On the contrary, MEK2 knockdown markedly inhibited the invasive capability of PC-1.0 cells compared with the control cells (invasive cell number $=20.1 \pm 3.1, \mathrm{P}<0.05$ ). However, down-regulation of MEK1 showed no significant inhibitory effect on the invasive capability of PC-1.0 cells (invasive cell number $=48.4 \pm 4.3, \mathrm{P}>0.05)$.

\section{Discussion}

MEK proteins contain regulatory sites where numerous signals can be integrated. Two regions of MEK1 and MEK2 show considerable divergence: the N-terminal ERK-binding site and the phosphorylation region, where several other signalling proteins bind. The differences in these important regions suggest MEK1 and MEK2 may not have fully redundant functions. This hypothesis is supported by studies of knockout mice that revealed functional differences between the two proteins $(16,17)$. There are also studies that indicate MEK1 and MEK2 perform specific functions in hepatocytes. MEK2 mediates survival, whereas MEK1 is involved in proliferation (18). In the present study, we knocked down MEK1 and MEK2 expression to examine their functions in the progression of pancreatic cancer. Although certain smallmolecule inhibitors of MEK1/2 (e.g., U0126) have been tested as potential candidates for cancer therapy $(5,19)$, most of the inhibitors target MEK1 and MEK2 simultaneously (20). Therefore, these inhibitors lack specificity. On the other hand, RNAi is considered a powerful tool to examine gene function and could form the basis of novel therapeutic strategies for treating human diseases, including cancer (21).

In this study, we demonstrated that MEK1 and MEK2 mediate different biological functions in pancreatic cancer cells. The results showed that inhibition of MEK1, but not MEK2 expression, was an effective and specific method of inhibiting cell proliferation and inducing $\mathrm{G}_{0} / \mathrm{G}_{1}$ arrest. Moreover, targeting MEK2 mRNA specifically disrupted cell morphology and reduced the invasive ability of cultured pancreatic cancer cells. Our findings suggest that recombinant retroviruses expressing shRNAs against MEK1 and MEK2 have the potential to serve as therapeutic agents in the treatment of pancreatic cancer.

\section{Acknowledgements}

This study was supported by a grant-in-aid from the National Nature Science Foundation of China (No. 30973501). We would like to acknowledge Professor Hideo Baba for the kind gift of the PC-1 and PC-1.0 cell lines.

\section{References}

1. Egami H, Takiyama Y, Cano M, Houser WH and Pour PM: Establishment of hamster pancreatic ductal carcinoma cell line (PC-1) producing blood group-related antigens. Carcinogenesis 10: 861-869, 1989.
2. Egami H, Tomioka T, Tempero M, Kay D and Pour PM: Development of intrapancreatic transplantable model of pancreatic duct adenocarcinoma in Syrian golden hamsters. Am J Pathol 138: 557-561, 1991.

3. Pour PM, Egami H and Takiyama Y: Patterns of growth and metastases of induced pancreatic cancer in relation to the prognosis and its clinical implications. Gastroenterology 100: 529-536, 1991.

4. Ishikawa S, Egami H, Kurizaki T, et al: Analysis of the factor in related to the signal transduction pathway of invasion and metastasis in pancreatic cancer. J Exp Clin Cancer Res 22: 299-306, 2003.

5. Tan X, Egami H, Kamohara H, et al: Involvement of the mitogen-activated protein kinase kinase 2 in the induction of cell dissociation in pancreatic cancer. Int $\mathrm{J}$ Oncol 24: 65-73, 2004.

6. Robinson MJ and Cobb MH: Mitogen-activated protein kinase pathway. Curr Opin Cell Biol 9: 180-186, 2003.

7. Cobb MH: MAP kinase pathways. Prog Biophys Mol Biol 71: 479-500, 1999.

8. Kolch W: Meaningful relationships: the regulation of the Ras/Raf/MEK/ERK pathway by protein interactions. Biochem J 351: 289-305, 2000.

9. Bryan $\mathrm{AB}$ and Blenis $\mathrm{J}$ : Molecular mechanisms mediating mammalian mitogen-activated protein kinase (MAPK) kinase (MEK)-MAPK cell survival signals. Cell Growth Differ 12: 397-408, 2000.

10. Schaeffer HJ and Weber MJ: Mitogen-activated protein kinases: specific messages from ubiquitous messengers. Mol Cell Biol 19: 2435-2444, 1999.

11. Eblen ST, Slack JK, Weber MJ and Catling AD: Rac-PAK signaling stimulates extracellular signal-regulated kinase (ERK) activation by regulating formation of MEK1-ERK complexes. Mol Cell Biol 22: 6023-6033, 2002.

12. Wu X, Noh SJ, Zhou G, Dixon JE and Guan KL: Selective activation of MEK1 but not MEK2 by A-Raf from epidermal growth factor stimulated Hela cells. J Biol Chem 271: 3265-3271, 1996.

13. Xu S, Khoo S, Dang A, et al: Differential regulation of mitogenactivated protein/ERK kinase (MEK)1 and MEK2 and activation by a Ras-independent mechanism. Mol Endocrinol 11: 1618-1625, 1997.

14. Liu L, Zhang Q, Zhang Y, Wang S and Ding Y: Lentivirusmediated silencing of Tiam I gene influences multiple functions of a human colorectal cancer cell line. Neoplasia 8: 917-924, 2006.

15. Tan X, Egami H, Ishikawa $\mathrm{S}$, et al: Involvement of matrix metalloproteinase-7 in invasion-metastasis through induction of cell dissociation in pancreatic cancer. Int J Oncol 26: 1283-1289, 2005.

16. Giroux S, Tremblay M, Bernard D, et al: Embryonic death of Mek1-deficient mice reveals a role for this kinase in angiogenesis in the labyrinthine region of the placenta. Curr Biol 9: 369-372, 1999.

17. Belanger, LF, Roy S, Tremblay M, et al: Mek2 is dispensable for mouse growth and development. Mol Cell Biol 23: 4778-4787, 2003.

18. Skarpen E, Flinder LI, Rosseland CM, et al: MEK1 and MEK2 regulate distinct functions by sorting ERK2 to different intracellular compartments. FASEB J 22: 466-476, 2008.

19. Tan X, Egami H, Ishikawa S, et al: Relationship between the expression of extracellular signal-regulated kinase $1 / 2$ and the dissociation of pancreatic cancer cells: Involvement of ERK1/2 in the dissociation status of cancer cells. Int J Oncol 24: 815-820, 2004.

20. Favata MF, Hriuchi KY, Manos EJ, et al: Identification of a novel inhibitor of mitogen-activated protein kinase kinase. J Biol Chem 273: 18623-18632, 2002.

21. Silva J, Chang K, Hannon GJ and Rivas FV: RNA-interferencebased functional genomics in mammalian cells: reverse genetics coming of age. Oncogene 23: 8401-8409, 2004. 